\title{
A Prospective, Randomised, Controlled Study on the Effect of Black Tea on Newly Detected Type 2 Diabetes
}

\author{
Anirban Majumder ${ }^{1}$, Debmalya Sanyal ${ }^{1 *}$, Soumyabrata Roy Chaudhuri ${ }^{1}$ and Somwrita Mitra ${ }^{2}$ \\ ${ }^{1}$ Department of Endocrinology, KPC Medical College and Hospital, India \\ ${ }^{2}$ Diploma in Dietetics and Certified Diabetes Educator, India
}

Submission: April 04, 2020; Published: May 05, 2020

*Corresponding author: Debmalya Sanyal, Professor, Department of Endocrinology, KPC Medical College and Hospital, India

Abstract

Tea is one of the most widely consumed beverages in the world and is known for its high polyphenol content that has various health benefits. This investigation aims to study the potential of black tea compounds on subjects with new onset Type 2 diabetes mellitus. For this, 30 subjects with newly detected type 2 diabetes mellitus will be randomly enrolled into the study and will be advised to consume black tea on daily basis in a stipulated quantity. Anthropometric measurements, systolic and diastolic blood pressure, glycaemic and lipid profiles along with creatinine values will be analyzed during the study period. The effects of regular black tea consumption will then be assessed by observing the changes in the biochemical parameters at the end of the scheduled 12th week visit. A positive result will help us to popularise black tea as a safe as also a beneficial beverage for diabetic patients.

Keywords: Type 2 Diabetes; Beverages; Anthropometric measurements; Systolic and diastolic blood pressure; Glycemic and lipid profiles; Diabetic patients; Metformin; Sulphonylureas; Insulin

\section{Introduction}

Type 2 diabetes, a chronic metabolic disorder, is rising alarmingly all over the world. According to the International Diabetes Federation 2015, out of 415 million diabetics worldwide, the number of people having diabetes in India was around 78.3 million and was expected to be doubled around 140.2 million by 2040 until and unless urgent preventive steps are taken [1]. Lots of studies show that the incidence of Diabetes Mellitus is reaching potentially epidemic proportions in India and the reason behind this is multifactorial which includes genetic factors, obesity associated with the economic boom, rising standard of living, the steady migration of people from rural to urban area, changes in dietary patterns and decreased physical activity [2]. Under this scenario the management of diabetes is becoming a challenge. Type 2 diabetes cases are treated by both medicinal as well as non-medicinal way. However, taking medicines like metformin, sulphonylureas, insulin on daily basis are expensive and have numerous side effects. Apart from weight gain, gastrointestinal disturbances, patients also suffer from hypoglycemia due to the imbalance between medicines, food and activity [3]. Currently tea has been found to possess widespread biological functions based on the result of various studies. The effects of tea on obesity and diabetes have received increasing attention of late [4].

\section{Review}

Diabetes Mellitus is a non-communicable metabolic disorder, had a place in Egyptian manuscripts dating back to 1500 B.C. The Indian physicians called it madhumeha or ' honey urine' because it attracted ants. The ancient Indian physician Sushruta and the surgeon Charaka identified the two types of diabetes (400-500 A.D.), later to be named as Type I and Type II diabetes [5]. In the new millennium this disease has become epidemic. India leads the world with second largest number of diabetic subjects till now due to increased insulin resistance, greater abdominal adiposity, lower adiponectin and higher high sensitive C-reactive protein levels. Other factors are genes, obesity, rising living standard, changes in dietary patterns (mostly refined foods) and decreased physical activity. If not treated early that leads to many complications both microvascular (neuropathy, nephropathy and retinopathy) and macrovascular (cardiovascular and peripheral vascular disease) with huge economic burden in the later life [68]. The greatest savings would be achieved only by preventing the progression of the complications. On the basis of numerous studies, dietary modifications and exercise has important role in controlling the disease $[9,10]$. 


\section{Current Research in Diabetes \& Obesity Journal}

Black tea is a product made from the Camellia sinensis plant; an evergreen shrub native to Asia. It contains the aflavin and the arubigins as major polyphenols reported to possess a broad spectrum of biological activities which includes antioxidant properties, reduction of various cancers, inhibition of inflammation, and protective effects against diabetes, hyperlipidemia and obesity [11]. In current researches, the positive effects of polyphenols on glucose homeostasis observed in a large number of in vitro and animal models. To confirm the implications of polyphenol consumption for prevention of insulin resistance, metabolic syndrome and type 2 diabetes, humantrials with well-defined diets, controlled study designs with holistic approaches are needed in our country [12]. Although there are many studies on the effect of green tea, the studies on the effect of black tea on Type 2 diabetes are lacking in India along with other Asian countries.

\section{Objective}

In our study we are trying to emphasize on impact of nonmedicinal natural compounds in early diabetes where both costing and side effects would be low. The moto of our study is to show the impact of the compounds of black tea on anthropometric indices, glycemic parameters, lipid profile and creatinine level on newly diagnosed Type 2 diabetes mellitus.

\section{Methodology}

A randomized controlled study is planned for being carried out at KPC Medical College and Hospital (KPCMC\&H) of South Kolkata, a multispeciality hospital having 750 beds that serves patients from all classes of the society. Among the registered diabetic subjects screened at the outdoor clinic on the basis of their blood reports, around 50 patients diagnosed recently with Type 2 diabetes and meeting the inclusion criteria will be enrolled for this study. The study is designed for 12 weeks or 3 months without medicine and supplemental food product (NICE guidelines).

The inclusion criteria include

a) Sex - Both male and female

b) Age -18 to 80 years

c) New onset Type 2 diabetes mellitus (Fasting plasma glucose $-\geq 126 \mathrm{mg} / \mathrm{dl}$ or Post prandial plasma glucose $\geq 200 \mathrm{mg} /$ dl or $\mathrm{HbA} 1 \mathrm{c}>6.5 \%$ )

d) $B M I-18$ to $35 \mathrm{~kg} / \mathrm{m}^{2}$

e) Willingness to participate in the study

f) Willingness to give consent

The exclusion criteria include

i. Male and female subjects $>80$ years age

\author{
ii. Pregnant and lactating woman \\ iii. Children $<18$ years age \\ iv. Type 1 diabetes \\ v. Admitted to hospital during the study period \\ vi. Taken anti-diabetic drug in past \\ vii. Having diabetic complications \\ viii. Consumption of tea $>8$ cups per day
}

After enrollment, the selected subjects will be conveyed about the study and an informed consent form will be given to them. After obtaining a written consent the subjects will be included in the study. After inclusion, selected subjects will be allocated to either of the two groups, A (test group) and B (control group). First group A consists of 30 subjects who will receive both life style modification and tea while the second group B consists of 20 subjects who will receive only lifestyle modification. This allocation will be done manually in 3:2 distributions for group A and group B respectively. Demographic data and anthropometric measurements will be taken from each group A and B after inclusion in the study and documented in the proforma for evaluation during the study period.

\section{Procedure of anthropometric measurements}

Body weight will be measured with a digital balance to the nearest $0.1 \mathrm{~kg}$, with light clothing. The height will be measured by using a wall-mounted stadiometer to the nearest of 0.1 $\mathrm{cm}$. The body mass index (BMI) will be calculated by using the equation weight $(\mathrm{kg}) /$ height $^{2}(\mathrm{~m})$. The waist circumference will be measured by an elastic meter to the nearest of $0.1 \mathrm{~cm}$. Blood pressure will be measured with a regular mercury manometer and the mean of two consecutive measurements, with a 15-minute interval, will be considered as the blood pressure of each subject.

Subjects from both group will be asked to do blood tests to examine FPG, PPPG, HbA1c, lipid profile, SGOT, SGPT, creatinine levels at the beginning and end of the study period, the values of which will be documented in the proforma. Both the groups A and $\mathrm{B}$ will be advised with standard lifestyle modifications as detailed below:

\section{The recommended lifestyle interventions include:}

Uniform calorie-based diet calculated according to Harris Benedict's Formulae, will be prescribed to all subjects as per ADA guidelines. This would include,

a. Replacing refined carbohydrates with wholegrain foods and increase intake of vegetables and other foods rich in dietary fiber

b. Reducing the amount of saturated fat in the diet

c. To perform at least $150 \mathrm{~min} /$ week of moderate-intensity 
aerobic physical activity (50 - $70 \%$ of maximum heart rate) at least 3 days /week with no more than 2 consecutive days without exercise.

\section{d. Abstinence from alcohol and smoking}

Additionally, the test group A will be advised with regular black tea consumption in a stipulated amount. Black tea of the same tea garden and the same lot will be obtained from Tea Board of India, Kolkata, West Bengal, India. This tea will be provided to all the subjects of group A (test group) on $1^{\text {st }}, 4^{\text {th }}$ and $8^{\text {th }}$ week during the study. An instruction sheet on the method of preparation of tea at home will be provided to them, so that they can read it if required. A lifestyle assessment sheet will also be distributed to every subject, which has to be filled at home during study period. Cups and teapot will also be provided from the study center in order to ensure consistency of the result.

Preparation of the sample at home

To maintain consistent results, the following steps will be recommended for preparation of black tea at home,

a. The pot should be borosilicate glass with a lid that fits loosely. It will be provided during study.

b. 2 gms of tea will be placed into the pot.

c. $200 \mathrm{ml}$ of drinking water will be allowed to boil in stainless steel bowl of $250 \mathrm{ml}$ volume for 5 minutes and allowed to cool for 20 seconds. Teflon coated cookware for boiling will be avoided.

d. $100 \mathrm{ml}$ of freshly boiling water will be poured into the pot

e. Brewing time will be 6 minutes

f. The brewed tea will be strained into borosilicate glass cup which will be provided during study.

g. Milk, sugar or any other additives, except lemon are not to be used during preparation.

Every subject from both groups will be contacted over phone and asked to come on $4^{\text {th }}, 8^{\text {th }}$ and $13^{\text {th }}$ week for compliance assessment. Black tea leaf sachets will be distributed and they have to bring back the leftovers during this period. All the biochemical parameters and anthropometric data will be evaluated thoroughly at the beginning and end of the 3month study. The results will be compared between the two groups. Subjects will have freedom to withdraw at any time.

\section{Details of the Study}

\section{0 week}

Among the subjects who attended the OPD along with their blood reports, the newly detected Type 2 Diabetes Mellitus subjects will be approached and if they express their willingness to participate then screening will be done and they will be included or excluded on the basis of the inclusion criteria. After screening, they will be enrolled and explained in detail about the study. After enrollment, an informed consent form will be given to them and they will be asked to discuss at home about the study. If they want to participate, then they have to sign the consent form and do blood tests to examine FPG, PPPG, HbA1c, lipid profile, SGOT, SGPT, creatinine levels from any NABL accredited laboratory and visit the OPD after 1 week.

\section{$1^{\text {st }}$ week}

After obtaining a written consent the subjects will be included in the study. After inclusion, the selected subjects will be divided into two groups - A (test group) and B (controlled group). Demographic details regarding name, address, phone number, age, sex, presence or absence of family history of diabetes along with the anthropometric measurements like height, weight, BMI, waist circumference will be taken into account from each group and documented in the proforma. Blood reports will be collected from them and biochemical parameters will be noted down in the proforma for evaluation during the study period.

Group A: Each of the subject of group A will be prescribed to drink $400 \mathrm{ml}$ (4 cups) lukewarm brewed black tea without milk and sugar 4 times per day (early morning, after breakfast, afternoon, evening) along with the given diet chart over and above his/her usual daily consumption of beverages. They will be instructed to maintain their dietary intake and regular physical activity throughout the study period. Lifestyle assessment sheets will be given, which has to be filled at home. An instruction sheet on the method of preparation of tea at home will be provided to them, so that they can read it if required. Cups, teapot will also be provided in order to ensure consistency of the result. They will be supplied with 4 sachets containing 2 gm of black tea leaf each per day or 56 sachets or 112 gms of black tea leaf for 2 weeks. An extra 16 sachets or 32 gms of black tea leaf will be also provided to avoid miss out of the dose in unavoidable circumstances. They will be asked to come on 4th week after the consumption of tea at home as per the instruction chart given to them.

Group B: Diet chart and lifestyle modification instruction will be given to the subjects of group B. The lifestyle assessment sheets will be given, which has to be filled at home. They will be asked to come on 4 th week after following the instructions at home.

\section{$2^{\text {nd }}$ week (telephonic visit)}

Subjects from both groups will be contacted by telephone. Reports on any adverse events will be noted down.

\section{$3^{\text {rd }}$ week (telephonic visit)}

Subjects from both groups will be contacted by telephone. 
They will be given a reminder for their next visit. Reports on any adverse events will be noted down. Group A subjects will be informed to bring back the leftover sachets. Both the groups will be asked to bring their diet chart and lifestyle assessment sheets.

\section{$4^{\text {th }}$ week}

Group A: Dietary counselling will be done and they will be supplied with 84 sachets or 168 gms of black tea leaf for 3 weeks. Replacement of extra sachet will be done, if required. The lifestyle assessment sheets will be given. They will be asked to come on 8th week. Reports on any adverse events will be noted down.

Group B: Dietary counselling will be done and the lifestyle assessment sheets will be given. They will be asked to come on 8th week. Reports on any adverse events will be noted down.

\section{$5^{\text {th }}$ week (telephonic visit)}

Telephonic conversation with both the groups. Reports on any adverse events will be noted down.

\section{$6^{\text {th }}$ week (telephonic visit)}

Telephonic conversation with both the groups. Reports on any adverse events will be noted down.

\section{$7^{\text {th }}$ week (telephonic visit)}

Telephonic conversation with both the groups. Reports on any adverse events will be noted down. They will be given a reminder for their next visit. Group A subjects will be informed to bring back the leftover sachets. Both the groups will be asked to bring their diet chart and lifestyle assessment sheets.

\section{$8^{\text {th }}$ week}

Group A: Dietary counselling will be done and they will be supplied with 112 sachets or 224 gms of black tea leaf for 4 weeks. Replacement of extra sachet will be done, if required. The lifestyle assessment sheets will be given. They will be asked to come on 13th week after doing the blood tests to reexamine FPG, PPPG, HbA1c, lipid profile, SGOT, SGPT \& creatinine levels from the same laboratory. Reports on any adverse events will be noted down.

Group B: Dietary counselling will be done and the lifestyle assessment sheet will be given. They will be asked to come on 13th week after doing the blood tests to reexamine FPG, PPPG, HbA1c, lipid profile, SGOT, SGPT \& creatinine levels from the same laboratory. Reports on any adverse events will be noted down.

\section{$12^{\text {th }}$ week (telephonic visit)}

Telephonic conversation with both the groups. Reports on any adverse events will be noted down. They will be given a reminder for their next visit. Group A subjects will be informed to bring back the leftover sachets. Both the groups will be asked to bring their lifestyle assessment sheets and to reexamine their blood after the completion of the week from the same laboratory. They will be asked to bring their blood reports on their next visit.

\section{$13^{\text {th }}$ week}

This is the last week and end of the study. Leftover sachets will be taken back from group A. Lifestyle assessment sheets will also be taken back from both the groups. The biochemical parameters of both the groups will be noted down from the blood reports and reassessment of anthropometric measurements will be done. All the data will be documented in the proforma.

After the end of the study each and every subject will be advised to continue their regular checkups at OPD.

Outcome measurements: At baseline and after 3months, the following will be assessed for both groups: Height, body weight, waist circumference, body mass index, blood pressure, fasting plasma glucose, post prandial plasma glucose, HbA1c, creatinine, lipid profile, SGOT and SGPT.

Total black tea intake will be calculated by,

Total black tea intake $=$ frequency of brewed black tea consumption (cups/ day) $\times$ portion size $(\mathrm{ml}) \times$ number of days

\section{Report of adverse events}

The patients will be strictly advised to immediately report any adverse effects as stomach upset, fever, allergy or any other related or unrelated to the study, within 24-hour span.

\section{Data analysis}

The data will be analysed using appropriate statistical methods to evaluate the significance of the results.

\section{Discussion}

An ecological study done in the year 2012 using a systematic data mining approach involving 50 different countries established a linear statistical correlation between high black tea consumption and low diabetes prevalence. Countries with highest black tea consumption as Ireland, Russia had the lowest occurrence of diabetes, while China with the lowest black tea consumption had the highest prevalence of diabetes [13]. The studies on the effect of black tea on Type 2 diabetes are lacking in India along with other Asian countries. Our study will be a small initiative to understand the effect of black tea on Type 2 Diabetes Mellitus, in India.

Our point of interest is on black tea because its health benefits have been overshadowed for a while by the growing fascination with the multitude of health benefits provided by green tea. Black tea, during processing, is oxidized more than other types of tea (green, white, oolong and pu-erh), resulting in very high levels of two types of antioxidants- theaflavins and thearubigins. Black tea's potential ability to prevent diabetes may be due to its unique oxidation process and distinctive antioxidants [13].

Tea including black tea contains caffeine and fluoride. Excessive consumption of these may lead to negative health effects. In order to minimize any risk relating to excess caffeine 
and fluoride consumption maximum black tea intake should not be more than 8 cups per day. Excess fluoride intake (>10 mg/ dl) may lead to skeletal fluorosis. In this study, subjects will be advised with daily consumption of 4 cups of black tea or fluoride levels equivalent to $0.8-1.6 \mathrm{mg}$ which is well below the safe level $[14,15]$. Longer brewing time also increases the fluoride level and teflon coated cookware should be avoided for boiling as it releases small amount of fluoride into boiling water [15]. So the brewing time should recommend to 6 minute in this study.

Adding milk to black tea has been shown to lead to polyphenolprotein complexes, which would compromise beneficial effects of black tea polyphenols [16], while adding sugar gives empty calories. So milk and sugar are avoided during the preparation of tea in this study. In addition to polyphenols, black tea also contains polysaccharides which are found to be more effective than the polysaccharides of green or oolong tea in slowing down the absorption of glucose from the intestine, which may help diabetics in managing the disease. The polysaccharides are also very effective in fighting free radicals which contribute to the development of diabetes [17]. and other chronic disorders. So drinking black tea may protect from developing diabetes.

\section{Conclusion}

Our study thus helps us to investigate whether black tea has any effect on newly diagnosed Type 2 diabetes and if the result will be positive, then it also help us to encourage a large scale and detailed investigation of the particular ingredient in black tea which may be responsible for such effect. The positive outcome of the study would help us to popularize black tea not only as a safe beverage for diabetic patients but also as a beverage of choice with long term health benefits. It could also be incorporated as part of the standard lifestyle modification protocol and recommended to newly diagnosed diabetic patients. This would obviously encourage regular black tea consumption and increase its potential in the global market.

\section{References}

1. Diabetes: facts and figures (2015) Source: IDF Diabetes Atlas Seventh Edition.

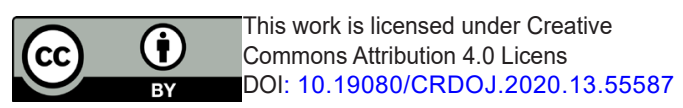

2. Mohan V, Sandeep S, Deepa R, Shah B, Varghese (2007) Epidemiology of type 2 diabetes: Indian scenario. Indian J Med Res 125(3): 217-230.

3. Itamar Raz (2013) Guideline Approach to Therapy in Patients with Newly Diagnosed Type 2 Diabetes. Diabetes Care 36(Suppl 2): S139-S144.

4. Hyun Min Kim, Jaetaek Kim (2013) The Effects of Green Tea on Obesity and Type 2 Diabetes. Diabetes Metab J 37(3): 173-175.

5. Vijay Viswanathan, Samriddhi Ranjan (2016) Diabetes - An ancient disease, epidemic $\&$ an economic burden for the present era. Indian J Med Res 143(4): 389-391.

6. Phillip Kantharidis, Bo Wang, Rosemarie M Carew, Hui Yao Lan (2011) Diabetes Complications: The MicroRNA Perspective. Diabetes 60(7): 1832-1837.

7. Brandle M, Zhou H, Smith BR, Marriott D, Burke R, et al. (2003) The direct medical cost of type 2 diabetes. Diabetes Care 26(8): 2300-2304.

8. Brown JB, Pedula KL, Bakst AW (1999) The progressive cost of complications in type 2 diabetes mellitus. Arch Intern Med 159(16): 1873-1880.

9. Wylie-Rosett J, Herman WH, Goldberg RB (2006) Lifestyle intervention to prevent diabetes: intensive and cost effective. CurrOpinLipidol 17(1): 37-44.

10. Mohammad Asif (2014) The prevention and control the type-2 diabetes by changing lifestyle and dietary pattern. J Educ Health Promot 3: 1.

11. Li S, Lo CY, Pan MH, Lai CS, Ho CT (2013) Black tea: chemical analysis and stability. Food Funct 4(1): 10-18.

12. Hanhineva K, Törrönen R, Bondia-Pons, Pekkinen J, Kolehmainen M, et al. (2010) Impact of dietary polyphenols on carbohydrate metabolism. Int J Mol Sci 11(4): 1365-1402.

13. Ariel Beresniak, Gerard Duru, Genevieve Berger, Dominique BremondGignac (2012) Relationships between black tea consumption and key health indicators in the world: an ecological study. BMJ Open 2(6): e000648.

14. E J Gardner, C H S Ruxton, A R Leeds (2007) Black tea - helpful or harmful? A review of the evidence. Eur J Clin Nutr 61: 3-18.

15. Izuora K1, Twombly JG, Whitford GM, Demertzis J, Pacifici R (2011) Skeletal fluorosis from brewed tea. J ClinEndocrinolMetab 96(8): 2318-2324.

16. van der Burg-Koorevaar MC, Miret S, Duchateau GS (2011) Effect of Milk and Brewing Method on Black Tea Catechin Bioaccessibility. J Agric Food Chem 59(14): 7752-7758.

17. Chen H, Qu Z, Fu L, Dong P, Zhang X (2009) Physicochemical properties and antioxidant capacity of 3 polysaccharides from green tea, oolong tea, and black tea. J Food Sci 74(6): C469-474.

Your next submission with Juniper Publishers
will reach you the below assets
- Quality Editorial service
- Swift Peer Review
- Reprints availability
- E-prints Service
- Manuscript Podcast for convenient understanding
- Global attainment for your research
- Manuscript accessibility in different formats
( Pdf, E-pub, Full Text, Audio)
- Unceasing customer service
Track the below URL for one-step submission
https://juniperpublishers.com/online-submission.php

